\title{
The Effect of Oxidation on the Far-Field Scattering of Aluminium Patch Antennae from Visible to UV
}

\author{
Duncan McArthur (iD) and Francesco Papoff $(\mathbb{D}$ \\ Department of Physics, SUPA, University of Strathclyde, 107 Rottenrow, Glasgow G4 0NG, UK
}

Correspondence should be addressed to Francesco Papoff; f.papoff@strath.ac.uk

Received 31 January 2019; Accepted 9 June 2019; Published 26 June 2019

Academic Editor: Giancarlo C. Righini

Copyright (c) 2019 Duncan McArthur and Francesco Papoff. This is an open access article distributed under the Creative Commons Attribution License, which permits unrestricted use, distribution, and reproduction in any medium, provided the original work is properly cited.

In this paper we evaluate the effect of oxidation on the total power scattered in the far field by a $60 \mathrm{~nm}$ radius $\mathrm{Al}$ sphere in the presence of a substrate comprised of either $\mathrm{Al}$ or silica $\left(\mathrm{SiO}_{2}\right)$. Using an effective medium approach to model the $\mathrm{Al}$ particle with an outer layer of alumina $\left(\mathrm{Al}_{2} \mathrm{O}_{3}\right)$, we find that the UV peak of total energy scattered in the far field shifts towards longer wavelengths for volume fractions of $\mathrm{Al}_{2} \mathrm{O}_{3}$ up to $20 \%$. When particles with these volume fractions are held above an $\mathrm{Al}$ substrate, enhancement of two orders of magnitudes of the far-field power radiated by a dipole in the gap can be observed. For larger volume fractions of $\mathrm{Al}_{2} \mathrm{O}_{3}$, the total intensity of light scattered is significantly reduced.

\section{Introduction}

In recent years a large number of papers in nanophotonics have been investigating the properties of aluminum because it is an abundant and low cost metal with plasmon modes in the visible and the ultraviolet [1-8]. Following earlier experiments [2], we have found $[9,10]$ that aluminum nanoparticles and substrates are ideally suited for label-free detection of weakly fluorescent molecules in the ultraviolet, because they have resonances with a much stronger far-field radiative enhancement than similar nanostructures of gold or silver, for wavelengths shorter than $370 \mathrm{~nm}$. In particular, Al nanostructures can significantly enhance the detection of fluorescence by emitters for which the emission rate is much less than the internal nonradiative decay rate [11] when the emitter is strongly coupled to electromagnetic modes that efficiently transport energy into the far field. By changing the size of the nanoparticle, resonances can be tuned between $150 \mathrm{~nm}$ and $650 \mathrm{~nm}$ and enhance, by orders of magnitude, both the far-field radiation and the decay rates of dipolar emitters placed in the gap between the nanoparticle and the substrate. This could have a profound impact on sensing of many important molecules that have radiative decays in the ultraviolet much weaker than nonradiative decays. Examples of such molecules are alkanes [12], most amino acids [13] in proteins and peptides, and DNA bases.

\section{Methods}

In order to evaluate the feasibility of these applications, it is important to consider in what way oxidation of the $\mathrm{Al}$ surfaces may affect the performance of this system. In this paper we investigate how the ratio between the power radiated above the substrate, $P_{r}$, and the total power emitted by the dipole in vacuum, $P_{0}$, depends on the volume fraction of alumina $\left(\mathrm{Al}_{2} \mathrm{O}_{3}\right)$ in the presence of a substrate of either silica $\left(\mathrm{SiO}_{2}\right)$ or Al. We consider substrates with thickness such that the light reflected by the lower surface is negligible, so that the substrates can be modeled as semi-infinite. The top layer of the Al substrate can also be oxidized, but for sake of simplicity we ignore here this effect, which should not change qualitatively the results presented when the oxidation layers are thin and do not support guided modes. We consider the following two configurations. The first configuration is similar to the standard set-up of a scanning near field optical microscope [14]: the particle lies on top of the substrate and 
it is illuminated by an electric dipole polarized along the axis $z$, which is perpendicular to the substrate, and placed $30 \mathrm{~nm}$ above the particle's surface. We investigate this configuration, even if it is not very suited to sensing, because it can be easily used to test the theory. The other configuration is the one most promising for sensing applications and is similar to patch antennae $[15,16]$. In this configuration, the particle is held $5 \mathrm{~nm}$ above the substrate and the dipole source is placed in the middle of the gap.

For all cases considered, we use the Bruggerman effective medium approximation [17] to model the effect of $\mathrm{Al}_{2} \mathrm{O}_{3}$ on the far-field scattering of light. The effective dielectric function, $\varepsilon$, for a material composed of $\mathrm{Al}$ and $\mathrm{Al}_{2} \mathrm{O}_{3}$ can be found, using given values of the permittivity for the two materials $[18,19]$ and their fractional volumes, from the following expression [20]:

$$
\frac{V_{\mathrm{Al}}}{V}\left(\frac{\varepsilon_{\mathrm{Al}}-\varepsilon}{\varepsilon_{\mathrm{Al}}+2 \varepsilon}\right)+\frac{V_{\mathrm{Al}_{2} \mathrm{O}_{3}}}{V}\left(\frac{\varepsilon_{\mathrm{Al}_{2} \mathrm{O}_{3}}-\varepsilon}{\varepsilon_{\mathrm{Al}_{2} \mathrm{O}_{3}}+2 \varepsilon}\right)=0,
$$

where $V=V_{\mathrm{Al}}+V_{\mathrm{Al}_{2} \mathrm{O}_{3}}$ is the total volume of the particle. For high volume densities of $\mathrm{Al}_{2} \mathrm{O}_{3}$, this theory has reproduced experimental results from $\mathrm{Al}$ nanodiscs [20] on $\mathrm{SiO}_{2}$ substrates more accurately than the Maxwell-Garnett theory [17].

The energy radiated above the substrate, $P_{r}$, is evaluated by integrating the far-field asymptotic form of the modulus squared of the electric field, given by Green's function, over the upper semispace. The electric field is calculated via an expansion in principal modes [21-25] of Green's function of the structure that includes the multiple scattering between the particles and the substrate at all orders $[9,10]$. The expansion of the scattering Green's function in terms of principal modes and their amplitudes is [10]

$$
G^{E P ; S}\left(\mathbf{r}, \mathbf{r}^{\prime}\right) \mathbf{p}\left(\mathbf{r}^{\prime}\right)=\sum_{n} a_{n}^{s}\left(\mathbf{p}\left(\mathbf{r}^{\prime}\right)\right) S_{n}^{E}(\mathbf{r}),
$$

where $S_{n}^{E}(\mathbf{r})$ is the electric field of the $n-t h$ principal mode at the point $\mathbf{r}$, and $a_{n}^{s}\left(\mathbf{p}\left(\mathbf{r}^{\prime}\right)\right)$ is the amplitude of $S_{n}^{E}$ due to the field generated by the dipole $\mathbf{p}$ in $\mathbf{r}^{\prime}$ (see Eq. (4)). The amplitude of each mode is the product of the "sensitivity" of the mode, which is an intrinsic property of the particle that depends only on the spatial correlation, at the surface of the particle, between the corresponding scattering and internal mode pairs, and the spatial correlation between these modes and the dipole field. This approach [9] can be applied to inhomogeneous host media [26] as long as nonlocal effects $[24,27]$ and quantum spill-out can be neglected [28].

In order to evaluate the aforementioned configurations, we solve Maxwell's equations for spherical particles above a semi-infinite substrate, with a monochromatic electric dipole source placed, either between or above the particle and the substrate, along the $z$ axis, which is the symmetry axis. The scattered field is expanded in terms of radiating electric and magnetic dipoles distributed inside the particle along the $z$-axis and polarized along the Cartesian axes. In order to consider self-consistently multiple scattering effects between particle and substrate, we add to each radiating dipole field the corresponding field reflected from the substrate calculated via Sommerfeld integrals $[29,30]$. For the internal field we use spherical multipoles, with origin at the center of the particle [31, 32].

With this choice of internal and scattered fields, we can form the principal modes as for a single particle in a homogeneous medium [21] and then use these modes in Eq. (2) to approximate Green's function. The procedure is as follows. First of all, we define the scalar product $f \cdot g=$ $\sum_{i=1}^{4} \int_{\partial V} f_{i}^{*} g_{i} \mathrm{~d} A$, with $f_{i}, g_{i}$ the tangent electric $(i=1,2)$ and magnetic $(i=3,4)$ components, in an arbitrary orthonormal system, of two solutions of Maxwell's equations at the surface of the particle $\partial V$. In order to improve the numerical stability, the electromagnetic field of each source is normalised so that $f \cdot f=1$. Orthonormal internal and scattering mode bases are formed via QR decompositions of the internal and scattered surface fields separately $[23,33]$, using freely available linear algebra routines [34]. Using these bases we then find principal internal, $\left\{i_{n}\right\}$, and scattered modes, $\left\{s_{n}\right\}$, such that $i_{n} \cdot s_{m}=\delta_{n m}$, with $\delta_{n m}$ the Kronecker delta. This is done numerically via singular value decomposition (SVD) [34] of the product of the internal and scattering orthonormal modes. For an incident field, the amplitudes of the internal principal modes are obtained by projecting the incident field along the scattered principal modes and vice versa. Hence, to evaluate Green's functions we have to apply an incident field, $\mathbf{F}^{0}$, radiated by a dipole. The mode amplitudes are then defined as

$$
\begin{aligned}
& a_{n}^{i}\left(\mathbf{p}\left(x^{\prime}\right)\right)=i_{n}^{\prime} \cdot \frac{\mathbf{F}^{0}}{\left[1-\left(s_{n} \cdot i_{n}\right)^{2}\right]} \\
& a_{n}^{s}\left(\mathbf{p}\left(x^{\prime}\right)\right)=-s_{n}^{\prime} \cdot \frac{\mathbf{F}^{0}}{\left[1-\left(s_{n} \cdot i_{n}\right)^{2}\right]}
\end{aligned}
$$

with $i_{n}^{\prime} \equiv i_{n}-\left(s_{n} \cdot i_{n}\right) s_{n}$ and $s_{n}^{\prime} \equiv s_{n}-\left(s_{n} \cdot i_{n}\right) i_{n}$. The principal modes are normalized so that $i_{n} \cdot i_{n}=s_{n} \cdot s_{n}=1$, $0 \leq i_{n} \cdot s_{n}=s_{n} \cdot i_{n}=\leq 1$, and $i_{n}^{\prime}$ is orthogonal to $s_{n}$, and $s_{n}^{\prime}$ is orthogonal to $i_{n}$ with $i_{n}^{\prime} \cdot i_{n}=s_{n}^{\prime} \cdot s_{n}=1-\left(s_{n} \cdot i_{n}\right)^{2}$. To evaluate the radiative properties of these modes in the far field, the asymptotic forms of the scattering sources [29], for $|\mathbf{r}| \longrightarrow \infty$, are substituted when constructing the principal mode fields in Eq. (2).

The procedure described here is general and can be applied to any particle in which the normal is defined at any point on the surface. For the azimuthally symmetric nanostructures considered here, numerical calculations are vastly sped up by forming internal and scattered fields whose tangent components have azimuthal angular dependence $\exp (i l \phi)$, with $l$ an integer number. For instance, fields with azimuthal dependence $\exp ( \pm i \phi)$ are obtained by combining dipoles distributed along the $z$ axis and (circularly) polarized along $x$ and $y$, while $z$ polarized dipoles distributed along the $z$ axis have fields that do not depend on $\phi$. This is suitable for the study of dipoles on the $z$ axis or close to it. We note, however, that, in order to study dipoles further away from the $z$ axis, sources with azimuthal angular dependence $\exp (i l \phi)$, with $|l|>1$, would be required. 


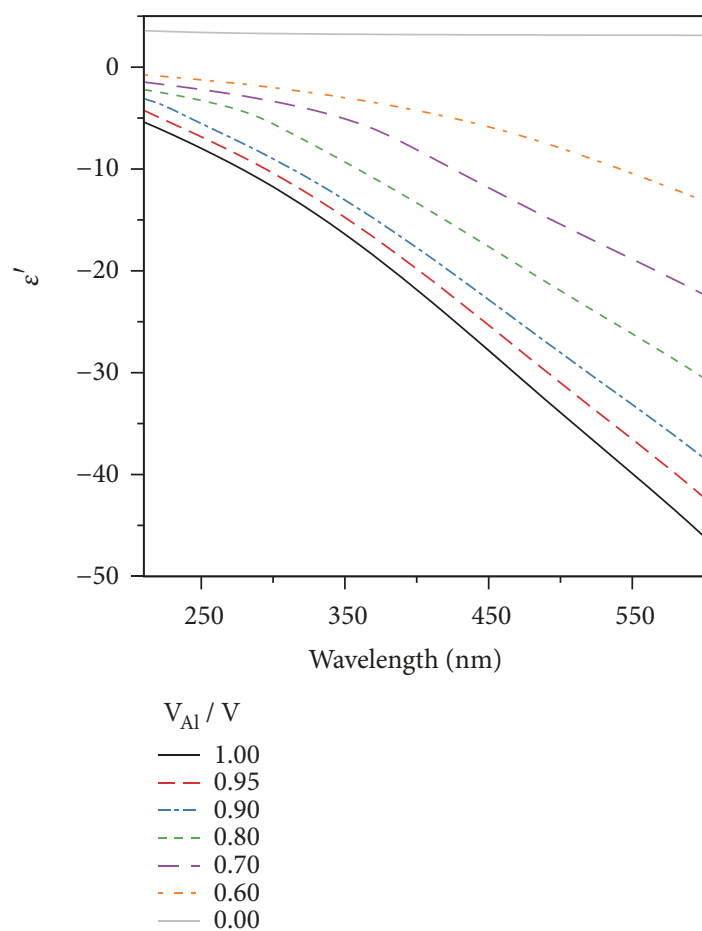

(a)

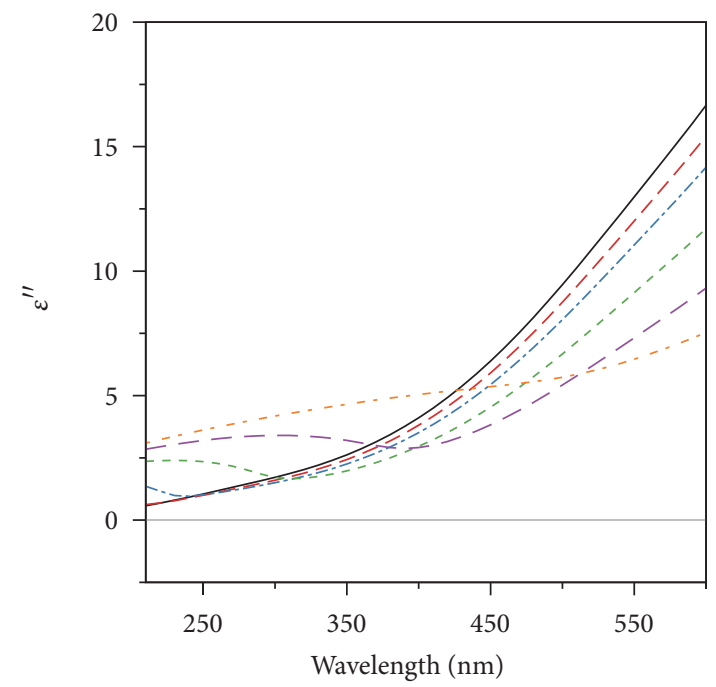

(b)

FIGURE 1: Complex dielectric function for $\mathrm{Al}+\mathrm{Al}_{2} \mathrm{O}_{3}$ calculated with the effective medium approach. For the complex permittivity, $\varepsilon=\varepsilon^{\prime}+i \varepsilon^{\prime \prime}$, we show the real, (a), and imaginary, (b), parts for particles with varying volume fractions of $\mathrm{Al}$, where 0 corresponds to pure $\mathrm{Al}_{2} \mathrm{O}_{3}$ and 1 to pure Al particles.

\section{Results and Discussion}

In Figure 1 we show the real and imaginary parts of the dielectric function, related to the refractive index and the absorption coefficient, respectively, for effective media with volume fractions of $\mathrm{Al}_{2} \mathrm{O}_{3}$ ranging from $5 \%$ to $40 \%$, as a function of the wavelength from $210-600 \mathrm{~nm}$. In the same figures we also show the refractive index and the absorption coefficient for pure $\mathrm{Al}$ and $\mathrm{Al}_{2} \mathrm{O}_{3}$. The effect of $\mathrm{Al}_{2} \mathrm{O}_{3}$ on the refractive index is straightforward: the higher the volume fraction is, the lower the absolute value of the refractive index is. From this, we can expect resonances to be shifted toward longer wavelengths as the volume fraction of $\mathrm{Al}$ decreases. The effect of $\mathrm{Al}_{2} \mathrm{O}_{3}$ on the absorption coefficient is more subtle and changes depending on the volume fraction and the wavelength. At longer wavelengths all volume fractions considered reduce the absorption coefficient with respect to the case of pure $\mathrm{Al}$. However, for $\mathrm{Al}$ volume fractions below $95 \%$, the absorption becomes higher at shorter wavelengths than for pure Al. The cross-over point where the effective medium absorption becomes higher than pure $\mathrm{Al}$ absorption moves toward longer wavelengths as the volume fraction of $\mathrm{Al}$ decreases. These features provide a qualitative explanation of the far-field response of spherical particles with different volume fractions.

In Figure 2 we compare the ratio $P_{r} / P_{0}$ for effective media spheres with radii of $60 \mathrm{~nm}$ lying on top of $\mathrm{Al}$ and $\mathrm{SiO}_{2}$ substrates. In both cases the UV peak in the scattered energy remains essentially unchanged for $\mathrm{Al}$ volume fractions of $90 \%$ or above. For an $\mathrm{Al}$ volume fraction of $80 \%$, the peak is reduced and moves further toward long wavelengths. However, for even lower Al volume fractions, the increased absorption reduces the peak and pushes it back towards shorter wavelengths.

In Figure 3 we show the ratio $P_{r} / P_{0}$ for the same effective media spheres held $5 \mathrm{~nm}$ above $\mathrm{Al}$ and $\mathrm{SiO}_{2}$ substrates, and with the dipole source placed in the middle of the gap. In this case the difference between the two substrates is much more significant. The ratio $P_{r} / P_{0}$ is an order of magnitude higher with the $\mathrm{Al}$ substrate and the corresponding enhancement in the far-field signal is of two orders of magnitudes for $\mathrm{Al}$ volume fractions higher than or equal to $80 \%$, with actually a slight increase in the peak value for the volume fractions $95 \%$ and $90 \%$ with respect to the pure $\mathrm{Al}$ case. This is accompanied by a shift towards longer wavelengths similar to what was observed in the previous case. However, the higher losses for lower $\mathrm{Al}$ volume fractions result in a suppression of the UV peak that is no longer distinguishable. With the $\mathrm{SiO}_{2}$ substrate, the ratio $P_{r} / P_{0}$ is an order of magnitude lower than with the Al substrate but the peaks remain distinguishable and move progressively toward longer wavelengths as the $\mathrm{Al}$ volume fraction is reduced.

\section{Conclusions}

In conclusion, we have presented an effective media study of Al particles with $\mathrm{Al}_{2} \mathrm{O}_{3}$ oxidation layers on top of, and above, substrates of $\mathrm{Al}$ and $\mathrm{SiO}_{2}$. The results can be qualitatively 


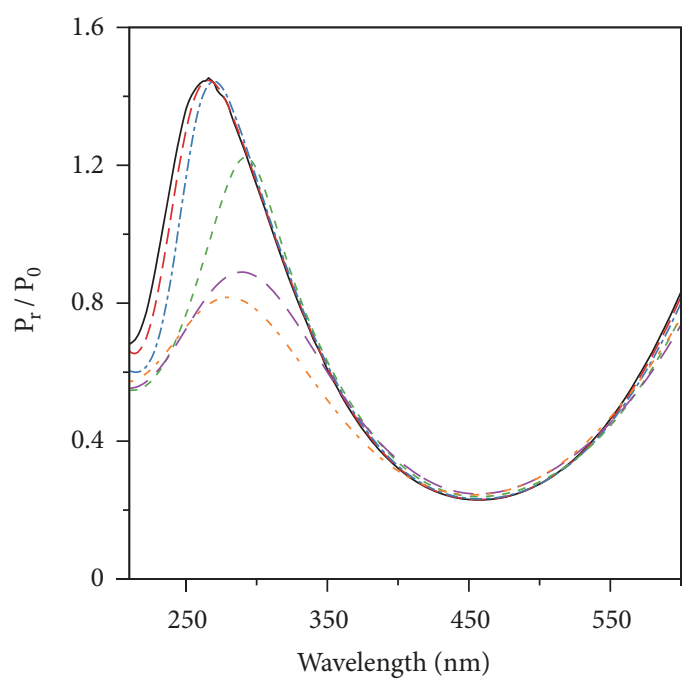

(a)

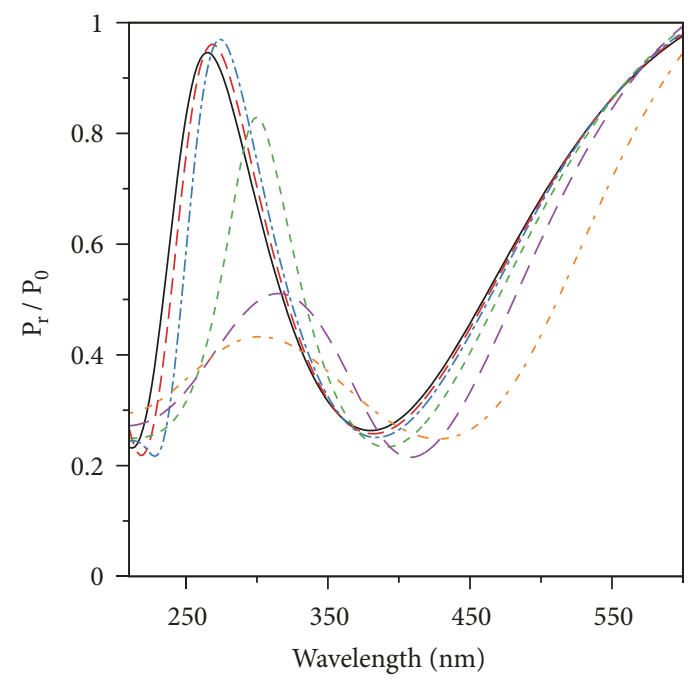

(b)

FIGURE 2: Normalised power radiated by spherical particles on top of a substrate, illuminated by an electric dipole. For substrates comprised of (a) $\mathrm{Al}$ and (b) $\mathrm{SiO}_{2}$. All curves correspond to the same volume fractions indicated in Figure 1 .

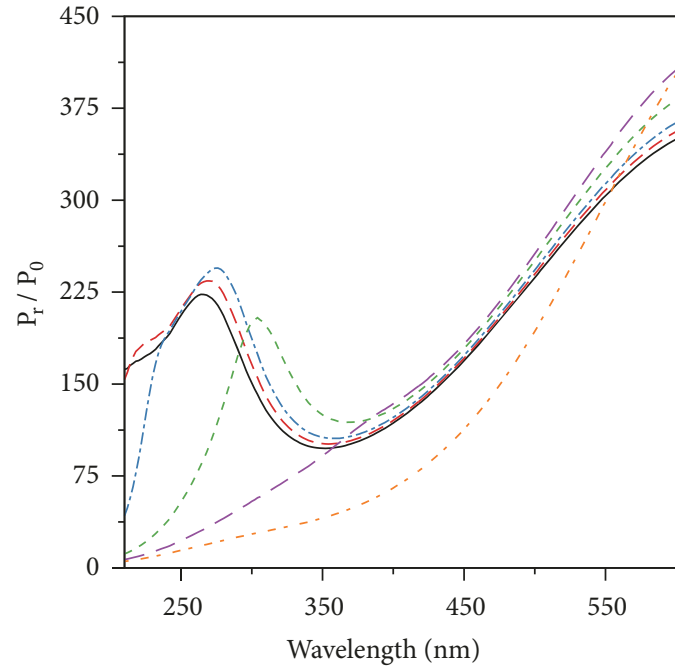

(a)

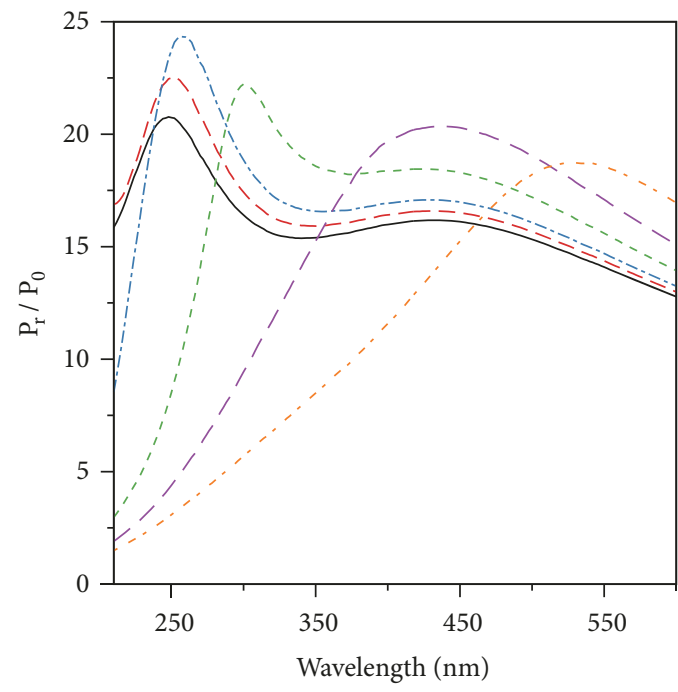

(b)

FIGURE 3: Normalised power radiated by spherical particles $5 \mathrm{~nm}$ above a substrate, illuminated by an electric dipole. For substrates comprised of (a) $\mathrm{Al}$ and (b) $\mathrm{SiO}_{2}$. All curves correspond to the same volume fractions indicated in Figure 1 .

explained by considering how the effective media absorption coefficient and refractive index depend on the volume fractions and the wavelengths. Our investigation of effective media spheres held above an $\mathrm{Al}$ substrate indicates that two orders of magnitude enhancement of the far-field radiation in the UV will take place also with oxidized $\mathrm{Al}$ particles as long as the volume fraction of $\mathrm{Al}$ is not below $80 \%$. In order to better appreciate the practical implication of these results, note that, for the $60 \mathrm{~nm}$ radius spheres considered here, the $95 \% \mathrm{Al}$ volume fraction corresponds to an outer layer of $\mathrm{Al}_{2} \mathrm{O}_{3}$ of $\sim 1 \mathrm{~nm}$, the $80 \% \mathrm{Al}$ volume fraction corresponds to $\sim 4.3 \mathrm{~nm}$ outer layer, and the $60 \% \mathrm{Al}$ volume fraction corresponds to $\sim 9.4 \mathrm{~nm}$ outer layer. These results, combined with the experimental findings [20] that the oxidation process in $\mathrm{Al}$ nanoparticles leads to stable layers of 3nm thickness, suggest that gap-enhanced spectroscopy is a viable option for the detection of weakly fluorescent molecules emitting in the UV. For more detailed investigations of the effect of oxidation on fluorescence, including the reduction of life time due to near field interaction with nonradiative modes, it will be necessary to use a more detailed model of the multilayered surfaces, as effective media theories have been tested mainly with respect to far-field properties.

\section{Data Availability}

The numerical data used to support the findings of this study are available from the corresponding author upon request. 


\section{Conflicts of Interest}

The authors declare that there are no conflicts of interest regarding the publication of this paper.

\section{Acknowledgments}

Duncan McArthur is funded by a Leverhulme Trust Research Grant.

\section{References}

[1] K. Ray, M. H. Chowdhury, and J. R. Lakowicz, "Aluminum nanostructured films as substrates for enhanced fluorescence in the ultraviolet-blue spectral region," Analytical Chemistry, vol. 79, no. 17, pp. 6480-6487, 2007.

[2] M. H. Chowdhury, K. Ray, S. K. Gray, J. Pond, and J. R. Lakowicz, "Aluminum nanoparticles as substrates for metalenhanced fluorescence in the ultraviolet for the label-free detection of biomolecules," Analytical Chemistry, vol. 81, no. 4, pp. 1397-1403, 2009.

[3] F. Mahdavi and S. Blair, "Nanoaperture fluorescence enhancement in the ultraviolet," Plasmonics, vol. 5, no. 2, pp. 169-174, 2010.

[4] X. Jiao and S. Blair, "Optical antenna design for fluorescence enhancement in the ultraviolet," Optics Express, vol. 20, no. 28, pp. 29909-29922, 2012.

[5] M. J. McClain, A. E. Schlather, E. Ringe et al., "Aluminum Nanocrystals," Nano Letters, vol. 15, no. 4, pp. 2751-2755, 2015.

[6] S. K. Jha, N. Mojarad, M. Agio, J. F. Löffler, and Y. Ekinci, "Enhancement of the intrinsic fluorescence of adenine using aluminum nanoparticle arrays," Optics Express, vol. 23, no. 19, pp. 24719-24729, 2015.

[7] N. S. King, L. Liu, X. Yang et al., "Fano Resonant Aluminum Nanoclusters for Plasmonic Colorimetric Sensing," Nano Letters, vol. 9, no. 11, pp. 10628-10636, 2015.

[8] D. Gérard and S. K. Gray, "Aluminium plasmonics," Journal of Physics D: Applied Physics, vol. 48, p. 184001, 2015.

[9] D. McArthur, B. Hourahine, and F. Papoff, "Enhancing ultraviolet spontaneous emission with a designed quantum vacuum," Optics Express, vol. 25, pp. 12189-12199, 2017.

[10] D. McArthur and F. Papoff, "Gap enhanced fluorescence as a road map for the detection of very weakly fluorescent emitters from visible to ultraviolet," Scientific Reports, vol. 7, p. 14191, 2017.

[11] M. Pelton, "Modified spontaneous emission in nanophotonic structures," Nature Photonics, vol. 9, no. 7, pp. 427-435, 2015.

[12] A. Volkmer, K. Wynne, and D. J. S. Birch, "Near-infrared excitation of alkane ultra-violet fluorescence," Chemical Physics Letters, vol. 299, pp. 395-402, 1999.

[13] C. D. McGuinness, K. Sagoo, D. McLoskey, and D. J. S. Birch, "A new sub-nanosecond LED at $280 \mathrm{~nm}$ : Application to protein fluorescence," Measurement Science and Technology, vol. 15, no. 11, pp. L19-L22, 2004.

[14] K. Imura, K. Ueno, H. Misawa et al., "Plasmon modes in single gold nanodiscs," Optics Express, vol. 22, no. 10, pp. 12189-12199, 2014.

[15] C. Sauvan, J. P. Hugonin, I. S. Maksymov, and P. Lalanne, "Theory of the Spontaneous Optical Emission of Nanosize Photonic and Plasmon Resonators," Physical Review Letters, vol. 110, p. 237401, 2013.
[16] R. Faggiani, J. Yang, and P. Lalanne, "Quenching, Plasmonic, and Radiative Decays in Nanogap Emitting Devices," ACS Photonics, vol. 2, no. 12, pp. 1739-1744, 2015.

[17] T. C. Choy, Effective Medium Theory: Principles and Applications, Oxford University Press, Oxford, UK, 1999.

[18] A. D. Rakić, A. B. Djurišić, J. M. Elazar, and M. L. Majewski, "Optical properties of metallic films for vertical-cavity optoelectronic devices," Applied Optics, vol. 37, no. 22, pp. 5271-5283, 1998.

[19] M. J. Dodge, "Refractive index," in Handbook of Laser Science and Technology, M. J. Weber, Ed., vol. IV of Optical Materials: Part 2, CRC Press, Boca Raton, Fla, USA, 1986.

[20] M. W. Knight, N. S. King, L. Liu, H. O. Everitt, P. Nordlander, and N. J. Halas, "Aluminum for plasmonics," ACS Nano, vol. 8, no. 1, pp. 834-840, 2014.

[21] F. Papoff and B. Hourahine, "Geometrical Mie theory for resonances in nanoparticles of any shape," Optics Express, vol. 19, no. 22, pp. 21432-21444, 2011.

[22] B. Hourahine and F. Papoff, "Optical control of scattering, absorption and lineshape in nanoparticles," Optics Express, vol. 21, no. 17, pp. 20322-20333, 2013.

[23] D. McArthur, B. Hourahine, and F. Papoff, "Evaluation of E. M. fields and energy transport in metallic nanoparticles with near field excitation," Physical Science International Journal, vol. 4, pp. 565-575, 2014

[24] D. McArthur, B. Hourahine, and F. Papoff, "Coherent control of plasmons in nanoparticles with nonlocal response," Optics Communications, vol. 382, pp. 258-265, 2017.

[25] B. Hourahine, D. McArthur, and F. Papoff, "Principal modes of Maxwell's equations," in The Generalized Multipole Technique for Light Scattering, T. Wriedt and Y. Eremin, Eds., vol. 99 of Springer Series on Atomic, Optical, and Plasma Physics, pp. 1-34, Springer International Publishing AG, 2018.

[26] W. Vogel and D.-G. Welsh, Quantum Optics, Wiley-VCH, Weinheim, Germany, 3rd edition, 2006.

[27] G. Toscano, J. Straubel, A. Kwiatkowski et al., "Resonance shifts and spill-out effects in self-consistent hydrodynamic nanoplasmonics," Nature Communications, vol. 6, p. 7132, 2015.

[28] W. Zhu, R. Esteban, A. G. Borisov et al., "Quantum mechanical effects in plasmonic structures with subnanometre gaps," Nature Communications, vol. 7, p. 11495, 2016.

[29] L. Novotny and B. Hecht, Eds., Principles of Nano-Optics, Cambridge University Press, 2nd edition, 2012.

[30] G. Y. Panasyuk, J. C. Schotland, and V. A. Markel, "Shortdistance expansion for the electromagnetic half-space Green's tensor: general results and an application to radiative lifetime computations," Journal of Physics A: Mathematical and Theoretical, vol. 42, no. 27, p. 275203, 2009.

[31] J. D. Jackson, Classical Electrodynamics, Wiley, New York, NY, USA, 1999.

[32] A. Doicu, Y. Eremin, and T. Wreidt, Acoustic and Electromagnetic Scattering Analysis Using Discrete Sources, Academic Press, 2000.

[33] G. H. Golub and C. F. Van Loan, Matrix Computations, Johns Hopkins University Press, Baltimore, Md, USA, 2nd edition, 1990.

[34] E. Anderson, Z. Bai, C. Bischof et al., LAPACK Users' Guide, Society for Industrial and Applied Mathematics, Philadelphia, $\mathrm{Pa}$, USA, 3rd edition, 1999. 

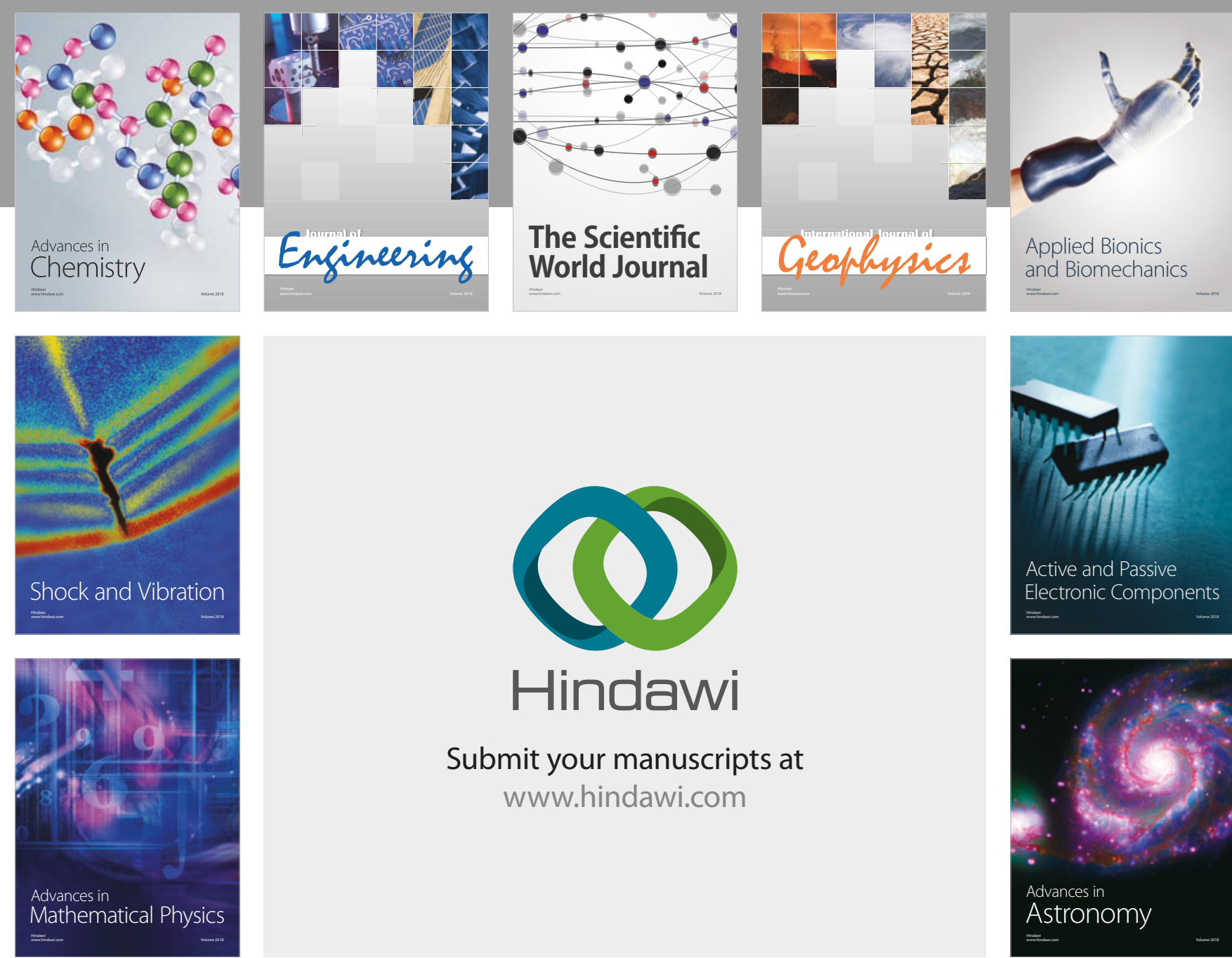

Submit your manuscripts at

www.hindawi.com

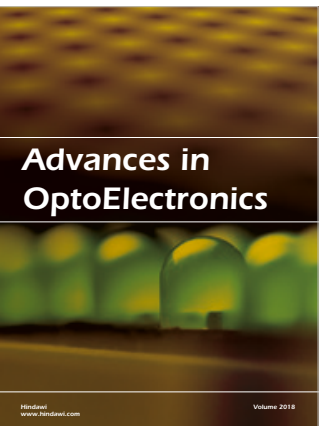

\section{Rotcting Machinery}
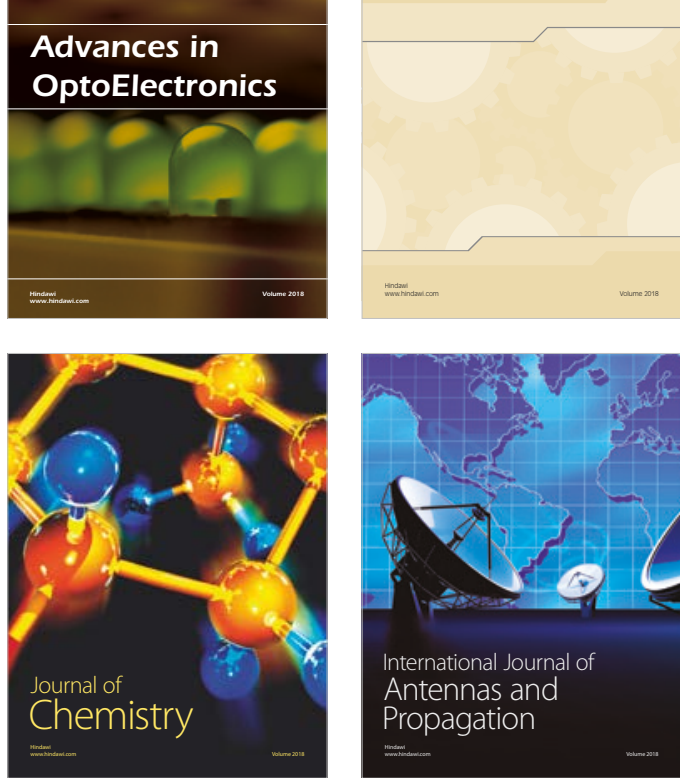

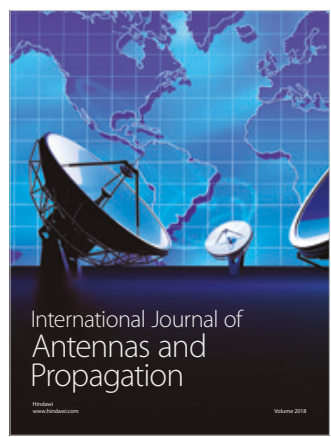

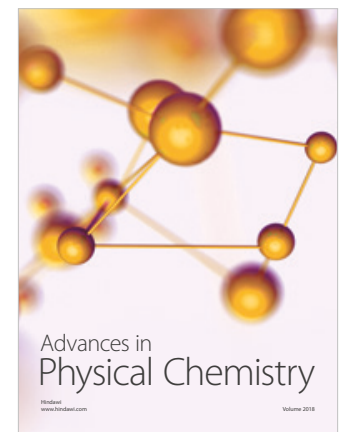

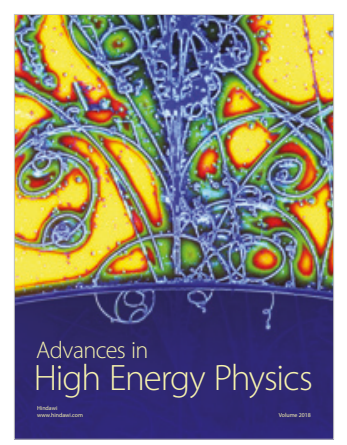

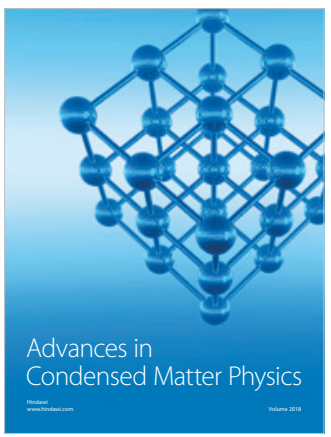

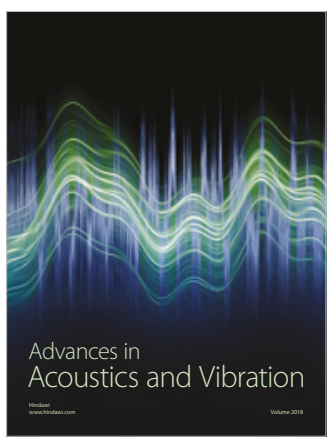

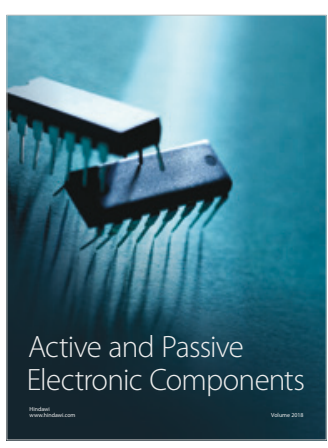
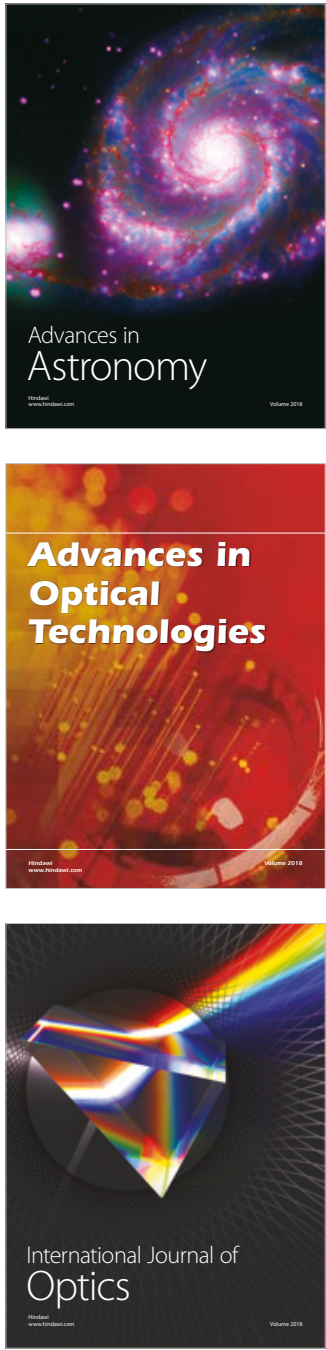